Research Journal of Pharmacology 13 (2): 27-31, 2019

ISSN: $1815-9362$

(C) Medwell Journals, 2019

\title{
Functional Group Analysis of Andrographis paniculata by FT IR Spectrum
}

\author{
${ }^{1}$ A. Doss and ${ }^{2}$ T.P. Kumari Pushpa Rani \\ ${ }^{1}$ Ethnopharmacology Unit, PG and Research Department of Botany, V.O. Chidamabaram College, \\ 628008 Tuticorin, India \\ ${ }^{2}$ Department of Microbiology, Kamaraj College, Tuticorin, Tamil Nadu, India
}

\begin{abstract}
The functional group analysis of various extracts (Hexane, Toluene, Tetrahydrofuran, Aqueous and Methanol) of potential indigenous medicinal plant Andrographis paniculata was recorded. The vibrational assignments, intensities and wave number $\left(\mathrm{cm}^{-1}\right)$ of dominant peak were obtained from absorption spectra. By this analysis, functional groups such as aminoacids, amides, amines, carboxylic acid, carbonyl compounds, organic hydrocarbons, halogens are present in all the 3 extracts. The present results showed that the crude extracts of this plant having high therapeutic value. In future it is used to treat against various diseases.
\end{abstract}

Key words: Functional groups, chemical compounds, medicinal plant, secondary metabolites, organic hydrocarbons, crude extracts

\section{INTRODUCTION}

Medicinal plants are the potential resource of drugs for ancient systems of medicine, therefore, man has been using crude plant extracts to protect himself against various diseases and also to improve his life-style. There are variety of secondary metabolites present in medicinal plants such as phenolic compounds, alkaloid, tannins, saponins, terpenoids, carboxylic acids, amino acids and inorganic acids. These secondary metabolites have wide array of medicinal properties (Parekh). Therefore, the analysis of these secondary metabolites would help in determining various biological activities of plants. A variety of techniques can be used to determine and estimate the presences of such phytocontituents in medicinal plants. Chromatography and spectroscopic techniques are the most useful and popular tools to detect these chemical compounds. FT-IR (Fourier Transform Infrared) spectral analysis of revealed the presence of various functional groups and chemical structures present in the bioactive constituents of medicinal plants (Maobe and Nyarango, 2013). The FT-IR analysis help to identify the alcohol, phenol, alkanes, alkyl halides, amino acids, carboxylic acid, aromatic, amines present in the medicinal plant extracts (Jagmohan, 2018). Moreover, FTIR spectroscopy is an established time saving method to characterize and identified functional groups (Grube).

\section{MATERIALS AND METHODS}

Collection of plant materials: The fresh leaves of $A$. paniculata were collected from the local areas. The collected plant material was authenticated by Dr. V. Nandagopalan, Associate Professor, PG and Research Department of Botany, National College, Tiruchirappalli, Tamilnadu. The voucher specimen has been deposited at the Department of Microbiology, Kamaraj College, Tuticorin, Tamil Nadu. The collected samples were air dried for 7 days at room temperature $\left(25^{\circ} \mathrm{C}\right)$. The dried samples were ground into fine powder and kept away from heat, moisture and sunlight.

Preparation of extracts: About $500 \mathrm{~g}$ dry powder of $A$. paniculata was sequentially extracted with hexane, toluene, tetrahydrofuran, methanol and water using the Soxhlet apparatus on the water bath for $12 \mathrm{~h}$ each. Each of the mixtures was carefully filtered using filter paper (Whatmann No. 1) and concentrated using a rotary evaporator. The extracts were stored in sterile bottles at $-18^{\circ} \mathrm{C}$ kept as aliquots until further evaluation.

Ultra Violet-Visible spectral analysis (UV-Vis): The electronic absorption spectra of the plant extracts were recorded in range of 200-800 nm in suitable solvent on a Systronics 2201 double beam UV-Vis., spectrophotometer. Absorbance values were plotted against the wave number. The electronic spectral measurements were used

Corresponding Author: A. Doss, Ethnopharmacology Unit, PG and Research Department of Botany, V.O. Chidamabaram College, 628008 Tuticorin, India 
for assigning the stereochemistry of ions in the complexes based on the number transition peaks (Saxena and Saxena, 2012).

\section{Fourier Transform Infra Red spectroscopy analysis (FT} IR): FT IR spectra of the plant extracts were recorded on Bruker Alpha T, Germany FT-IR 783 spectrophotometer in the range of $4000-550 \mathrm{~cm}^{-1}$ range using $\mathrm{KBr}$ disc $(\mathrm{KBr}$ pellet technique). The percentage transmission was recorded against wave number (Kabra et al., 2013). The peak values of the UV-VIS and FT IR were recorded.

\section{RESULTS AND DISCUSSION}

Spectroscopic techniques have powerful analytical tools for the analysis of pharmaceuticals and biological activities. The FT-IR spectrum was used to identify the functional groups of the active compounds based on the peak value in the region of infrared radiation. The different crude extracts of $A$. paniculata were analyzed by the FT IR spectrometers and the functional groups $f$ the compounds were separated based on its peak ratio.

The UV visible profile of the different extracts of Andrographis paniculata was elucidated at the wavelength of 200- $800 \mathrm{~nm}$ (Chanda et al., 2013) (Fig. 1). The different extracts of $A$. paniculata, showed the major bands at 290-300, 420-440 and $640 \mathrm{~nm}$ with the absorbance value of $2.35,2.279,2.406,2.349$ and 3.378, respectively. This showed the presence of phenols, flavonoids, carotenoids and chlorophylls. In another study reported that the UV-Visible spectra of 6 medicinal plants identified the maxima wavelengths specific for phenolics at $280-330 \mathrm{~nm}$, carotenoids at $420-470 \mathrm{~nm}$ and chlorophylls at $663 \mathrm{~nm}$ (Zavoi et al., 2011).

In the present study, hexane extract of $A$. paniculata bands occurring at 2967, 2866, 1661, 1377 and $1034 \mathrm{~cm}^{-1}$ corresponding to $\mathrm{C}-\mathrm{H}$ and $\mathrm{C}-\mathrm{O}$ stretching indicated the presence of alkanes and ether group of compounds (Fig. 2). The toluene extract of $A$. paniculata, the bands occurring at $3026,1603,1494$ and $1460 \mathrm{~cm}^{-1}$ corresponding to $\mathrm{N}-\mathrm{O}$ and $\mathrm{C}-\mathrm{O}$ stretching indicated the presence of amines, carboxylic group and ether group of compounds (Fig. 3). In an earlier study it was reported that the toluene extract of Semecarpus anacardium showed the bands at $3649,2918,1726$, 1315,1188 and $970 \mathrm{~cm}^{-1}$ corresponding to $\mathrm{O}-\mathrm{H}, \mathrm{C}-\mathrm{H}, \mathrm{C}-\mathrm{O}$, $\mathrm{NO}_{2}$ and $\mathrm{C}-\mathrm{H}$ stretching indicated the presence of

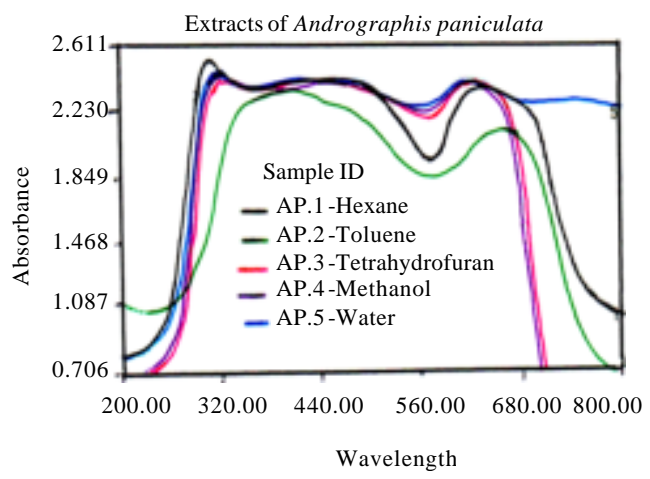

Fig. 1: UV spectrum of extracts of $A$. paniculata

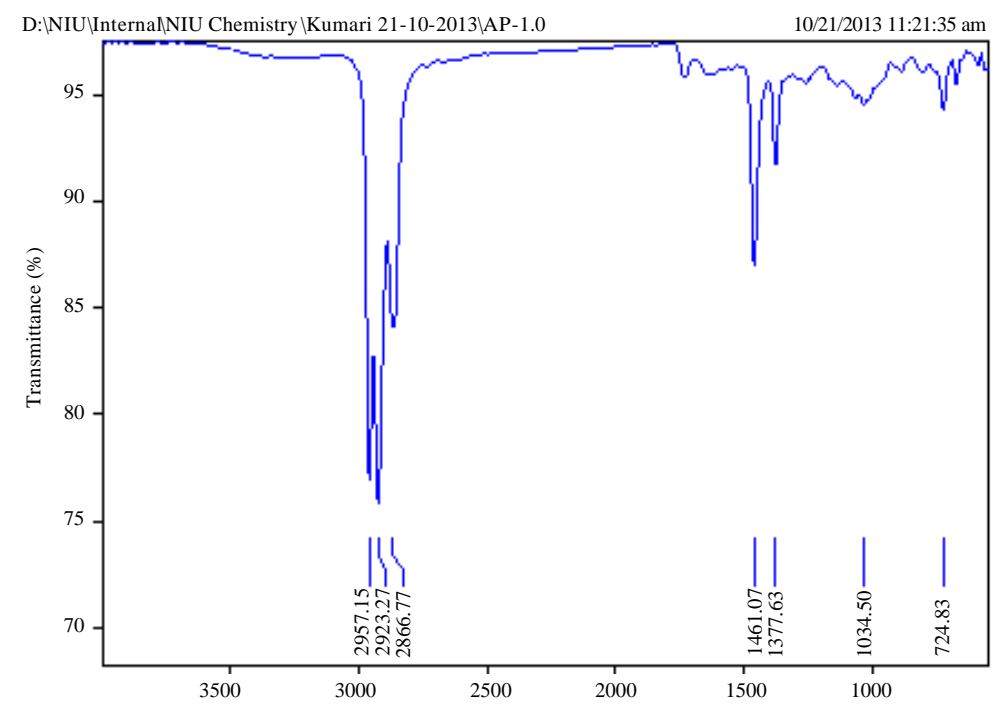

Fig. 2: FT IR spectrum of hexane extract of Andrographis paniculata 


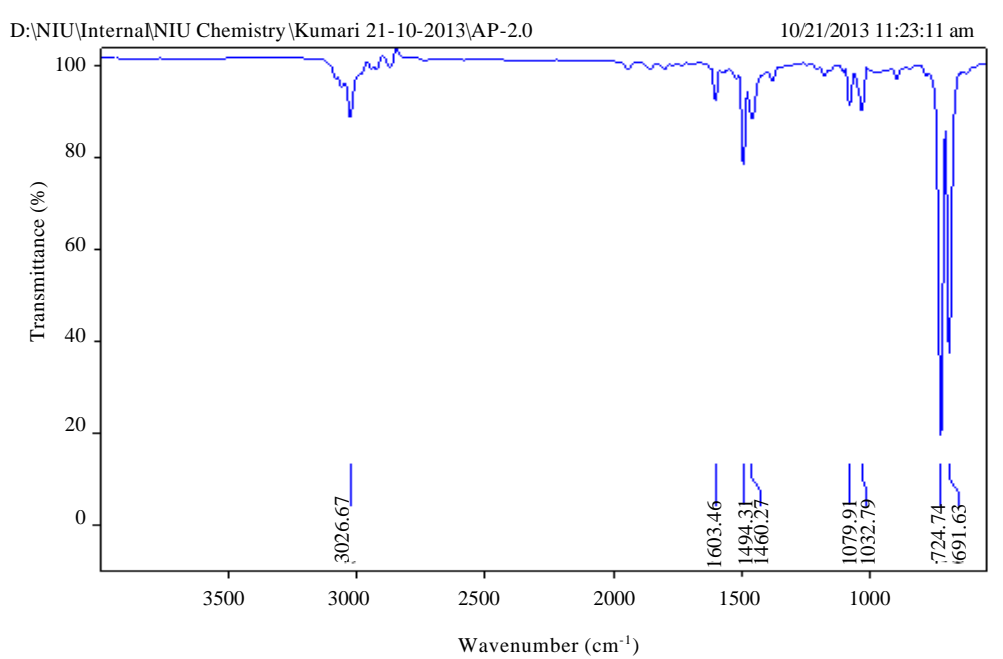

Fig. 3: FT IR spectrum of toluene extract of Andrographis paniculata

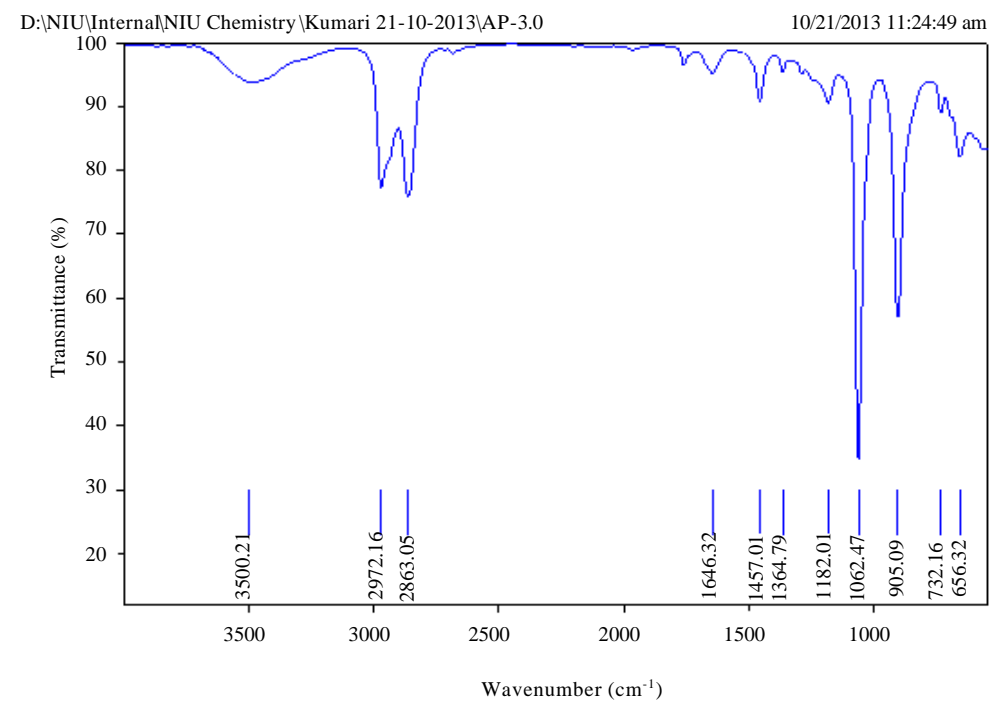

Fig. 4: FT IR spectrum of tetrahydrofuran extract of Andrographis paniculata

carboxylic acids, alkanes, aldehydes, nitro compound, secondary amines and alkene compounds (Pednekar and Raman, 2013).

In the present study, tetrahydrofuran extract of A. paniculata showed the bauds at $3500,3972,2863,1646$, 1457 and $1364 \mathrm{~cm}^{-1}$ corresponding to $\mathrm{OH}, \mathrm{C}-\mathrm{H}, \mathrm{C}=\mathrm{O}$ and $\mathrm{C}-\mathrm{O}$ stretching indicated the presence of alcohol, alkanes, aldehyde and ether group of compounds (Fig. 4). The methanol extracts showed the bands at 3436, 2997, 2914 , 1659 and $1434 \mathrm{~cm}^{-1}$ corresponding to $\mathrm{O}-\mathrm{H}, \mathrm{H}-\mathrm{O}$, $\mathrm{CH} / \mathrm{COOH}, \mathrm{C}=\mathrm{O}$ and $\mathrm{C}-\mathrm{O}$ stretching indicated the presence of hydroxyl, amines, alkanes, ketone and ether group of compounds (Fig. 5).

The aqueous extracts showed 3409, 2928, 21 47, 1704 and $1361 \mathrm{~cm}^{-1}$ corresponding to $\mathrm{O}-\mathrm{H}, \mathrm{H}-\mathrm{O}, \mathrm{C}=\mathrm{O}$ and $\mathrm{C}-\mathrm{O}$ stretching indicated the presence of hydroxyl, amines, ketone and ether group of compounds (Fig. 6). In an earlier report it was found that the methanol extract of Agar woods leaves showed the bands at 3384, 2923, $2851,1708,1618$ and $1468 \mathrm{~cm}^{-1}$ corresponding to $\mathrm{O}-\mathrm{H}$, $\mathrm{C}-\mathrm{H}, \quad \mathrm{C}=\mathrm{O}, \mathrm{C}=\mathrm{C}$ and $\mathrm{C}-\mathrm{C}$ stretching indicated the presence of alcohol, methylene, carboxylic acid and aromatic compounds (Khalil et al., 2013). More functional groups were recorded in the methanolic extract of the $A$. paniculata. The presence of characteristic functional groups carboxylic acids, amines, amides, sulphur derivatives, polysaccharides, organic hydrocarbons, halogens are responsible for various medicinal properties of Andrographis paniculata (Table 1). 


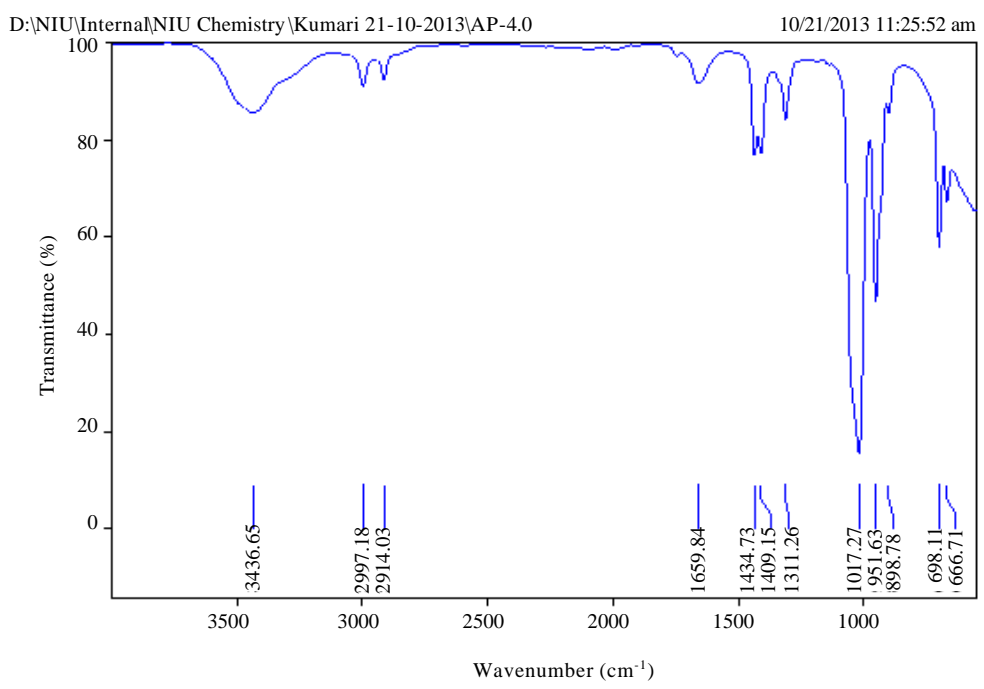

Fig. 5: FT IR spectrum of methanol extract of Andrographis paniculata

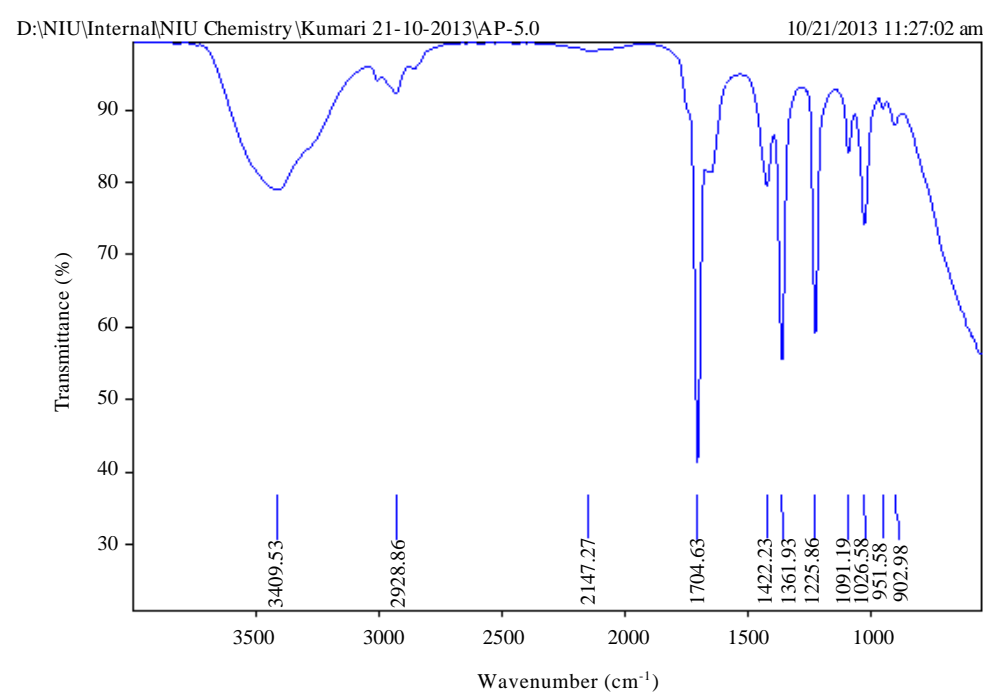

Fig. 6: FT IR spectrum of aqueous extract of Andrographis paniculata

Table 1: Various medicinla properties

\section{Wave number}

$\left(\mathrm{cm}^{-1}\right)$

3409

2928

2147

1704

$\underline{1361}$

\begin{tabular}{ll} 
Bond & Functional groups \\
\hline O-H & Hydrogen bonded alcohols, phenols \\
H-O & \\
C = O & Aldehydes, ketones, carboxylic acids, esters \\
C-O & Alcohols, ethers, carboxylic acids, esters \\
C-O & Alcohols, ethers, carboxylic acids, esters \\
\hline
\end{tabular}

\section{CONCLUSION}

The present studies, the functional groups present in the phytochemical constituents of the selected medicinal plant were elucidated using FT IR analysis and also, using for the treatment of various ailments.

\section{REFERENCES}

Chanda, S., Y. Baravalia and K. Nagani, 2013. Spectral analysis of methanol extract of Cissus quadrangularis L. stem and its fractions. J. Phoog. Phytochem., 2: 132-140.

Jagmohan, 2018. Organic Spectroscopy Principles and Applications. Narosa Publishing House, Delhi, India, Pages: 127.

Kabra, S., S. Katara and A. Rani, 2013. Characterization and study of Turkish perlite. Intl. J. Innovative Res. Sci. Eng. Technol., 2: 4319-4326.

Khalil, A.S., A.A. Rahim, K.K. Taha and K.B. Abdallah, 2013. Characterization of methanolic extracts of agarwood leaves. J. Appl. Ind. Sci., 1: 78-88. 
Maobe, M.A.G. and R.M. Nyarango, 2013. Fourier transformer infra-red spectrophotometer analysis of Urtica dioica medicinal herb used for the treatment of diabetes, malaria and pneumonia in Kisii region, Southwest Kenya. World Appl. Sci. J., 21: 1128-1135.

Pednekar, P.A. and B. Raman, 2013. The FT-IR spectrometric studies of vibrational bands of Semecarpus anacardium Linn. F. leaf, stem powder and extracts. Asian J. Pharm. Clin. Res., 6: 159-198.
Saxena, M. and J. Saxena, 2012. Evalution of phytoconstituents of Acorus calamus by FTIR and UV-VIS spectroscopic analysis. Intl. J. Biol. Pharm. Res., 3: 498-501.

Zavoi, S., F. Fetea, F. Ranga, A. Baciu and R.M. Pop et al., 2011. Comparative fingerprint and extraction yield of medicinal herb phenolics with hepatoprotective potential as determined by UV-Vis and FT-MIR spectroscopy. Notulae Botanicae Horti Agrobotanici Cluj Napoca, 39: 82-89. 